\title{
Characteristics and outcomes of patients with breast cancer diagnosed with SARS-Cov-2 infection at an academic center in New York City
}

\author{
Kevin Kalinsky ${ }^{1}\left[\right.$ - Melissa K. Accordino ${ }^{1} \cdot$ Kristina Hosi $^{1}$ - Jessica E. Hawley ${ }^{1}$ Meghna S. Trivedi ${ }^{1}$. \\ Katherine D. Crew ${ }^{1}$. Dawn L. Hershman ${ }^{1}$
}

Received: 23 April 2020 / Accepted: 5 May 2020 / Published online: 14 May 2020

(c) Springer Science+Business Media, LLC, part of Springer Nature 2020

Previous reports of patients with Coronavirus disease 2019 (COVID-19) showed higher severity of disease in cancer patients, including the intubation rate [1]. These series focused on those with symptoms from COVID-19 requiring hospitalization, with lung cancer being the most frequent malignancy $[1,2]$. We report characteristics and outcomes of COVID-19 + patients with breast cancer (BC) at an academic center in New York City.

\section{Methods}

We collected demographic, treatment, and outcome data from established patients with stage I-IV BC at Columbia University Irving Medical Center (CUIMC) and COVID$19+$ from $3 / 10 / 20$ to $4 / 29 / 20$ (data cut off). COVID-19+ was determined by reverse transcription-polymerase chain reaction (RT-PCR) by nasal swab and/or high clinical or radiographic suspicion. Initially, all outpatients with symptoms were referred to the emergency department (ED) for testing; however, outpatient testing, including at an ambulatory referral-based CUIMC fever clinic, was rapidly expanded with cobas ${ }^{\circledR}$ SARS-CoV-2 testing (Roche). This study was CUIMC Institutional Review Board approved.

\section{Results}

Of the 4515 COVID- $19+$ total patients at CUIMC, 27 $(0.6 \%)$ were established patients with a history of BC (Table 1). Twenty-six (96\%) were female; 15 (56\%) White, $6(22 \%)$ Black, and 12 (44\%) identified as Hispanic. The

Kevin Kalinsky

kk2693@cumc.columbia.edu

1 Columbia University Irving Medical Center, New York, NY, USA median age was 56 years (range: $32-87$ ), median body mass index was $28.5 \mathrm{~kg} / \mathrm{m}^{2}$ (range: $21-47$ ), and 7 (26\%) were current/former smokers. Co-morbidities included 15 (56\%) with hypertension, 6 (22\%) diabetes, and 6 (22\%) pulmonary disease. The majority had stage I-III BC and 5 (19\%) metastatic disease. In the 6 months prior to COVID-19+, 16 (59\%) received chemotherapy, 12 (44\%) hormone therapy, 6 (22\%) HER2-directed therapy, 1 (4\%) checkpoint inhibitor, 6 (22\%) breast surgery, and $2(7 \%)$ radiation therapy. Prior to COVID-19+, the last received therapy was chemotherapy for 14 patients [52\%, median: 12 days (range 7-749)], 10 (37\%) hormone therapy [median: 1 day (range 1-81)], and 3 (11\%) HER2-directed antibodies without chemotherapy [median: 21 days (range 20-34)]. Treatment disruptions occurred in 20 patients (74\%) due to COVID-19+.

Most common symptoms were cough (70\%), fever (52\%), shortness of breath (52\%), fatigue (30\%), diarrhea (22\%), and myalgia (19\%), with $30 \%$ having $\geq 4$ symptoms (Fig. 1). Twenty-two patients (81\%) had COVID-19+ confirmation by RT-PCR, 4 (15\%) presumed COVID-19+ per clinical symptoms, and 1 (4\%) based on imaging and symptoms. Eight (30\%) were initially tested in the ED, of whom 3 were not admitted. The majority were tested in the outpatient setting: ambulatory oncology clinic (6 patients; $22 \%$ ) or CUIMC fever clinic (8 patients: $30 \%$ ).

Of the 7 patients requiring hospitalization (26\%), 3 were non-Hispanic Black, 3 Hispanic White, and 1 non-Hispanic White. Two received recent chemotherapy without targeted agents for early stage BC and 5 single-agent hormone therapy ( 1 for metastatic disease). Five admitted patient had at least 1 co-morbid disease, and 3 were former smokers. Five admitted patients required supplemental oxygen, and none needed intensive care-level support, including intubation or dialysis. All were discharged from the hospital. With a median follow-up from COVID-19 + diagnosis of 26 days (range 1-38), all patients were alive, except for an 87-yearold male with coronary artery disease, hypertension, and 


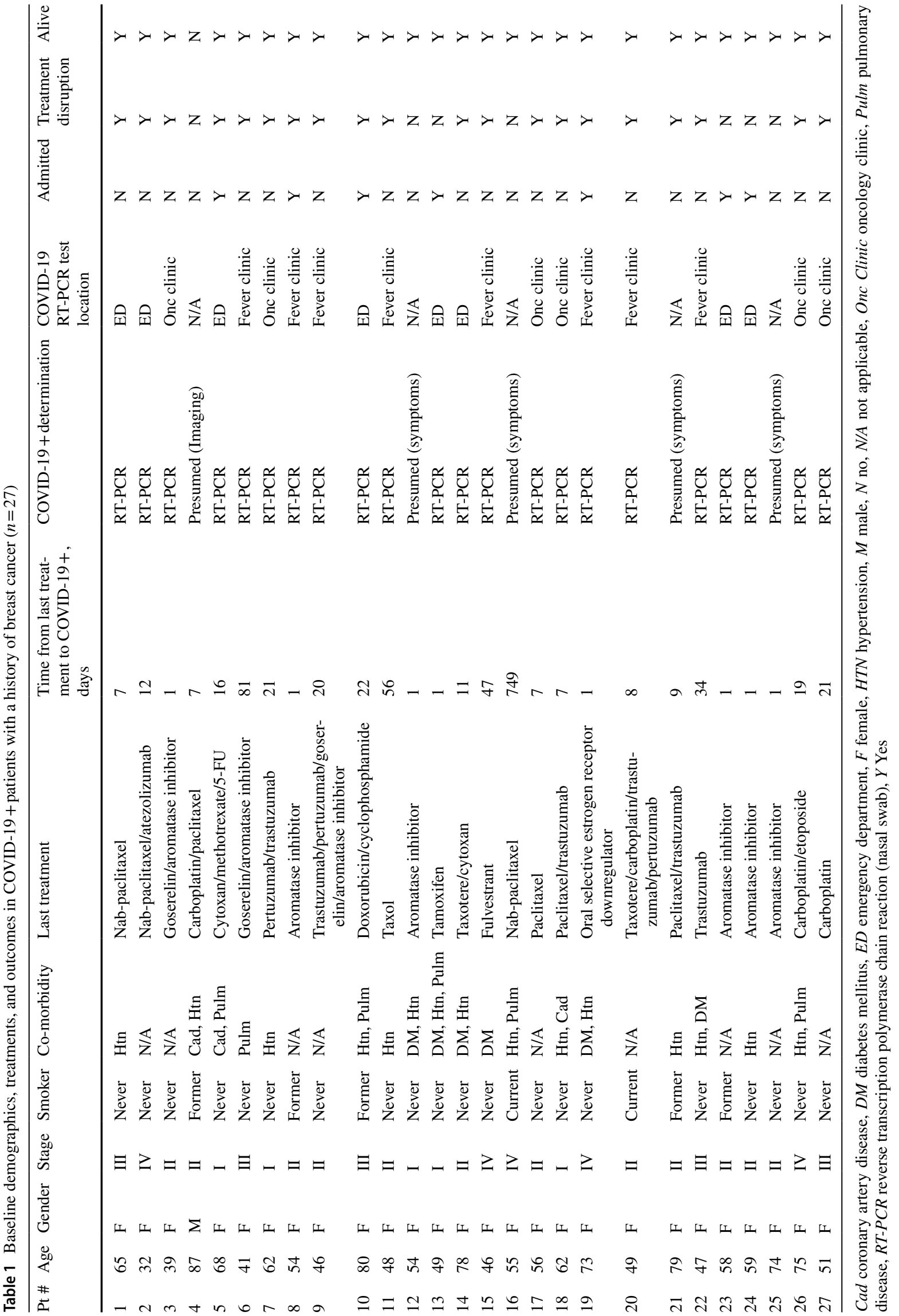



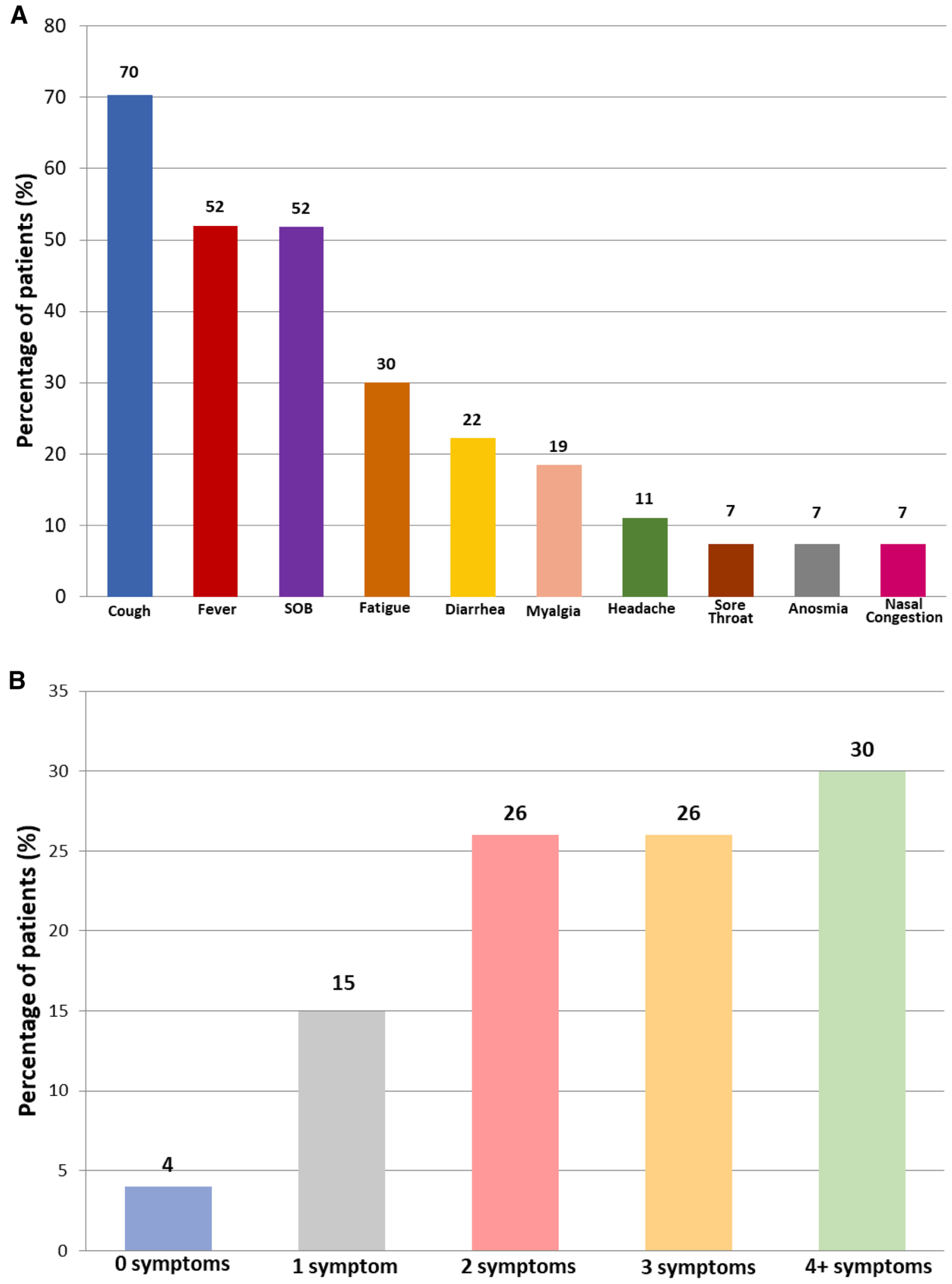

Fig. 1 a Percentage of COVID-19-related symptoms with which patients presented who had a history of stage I-IV breast cancer ( $n=27)$. b Percentage of these patients who presented with $0,1,2,3$, or at least 4 COVID-19-related symptoms 
former smoker who received taxane-based chemotherapy for stage II BC seven days before symptoms.

\section{Discussion}

In our racially/ethnically diverse population of 27 COVID$19+$ patients with BC, the majority $(74 \%)$ did not require hospitalization, and one male with multiple co-morbidities died. This is of interest, as COVID-19+ males have reported worse outcomes than females [3]. While treatment disruptions occurred in most patients (74\%), it is unknown whether this represents a deviation from other patients who develop infections on therapy. While larger series are needed to understand the impact of COVID-19 in patients with BC, these initial data are reassuring that a substantial number recover from their infection.

Funding No funding to report.

\section{Compliance with ethical standards}

Conflict of interest No conflicts are related to this particular study. For unrelated conflictions, Kevin Kalinsky served as a consultant/advisory board member for Immunomedics, Biotheranostics, Pfizer, Novartis, Eisai, Eli-Lilly, Amgen, and AstraZeneca. He has received institutional grants from Immunomedics, Novartis, Incyte, Genentech/Roche, Eli-Lilly, Pfizer, Calithera Biosciences, Acetylon, Seattle Genetics,
Amgen, Zentalis Pharmaceuticals, and CytomX Therapeutics. Served on speakers' bureau for Eli-Lilly. Received travel expenses from Lilly and Astra Zeneca. Spouse was previously employed by Novartis, Array Biopharma, and Pfizer.

Ethical approval All procedures performed in studies involving human participants were in accordance with the ethical standards of the institutional and/or national research committee and with the 1964 Helsinki declaration and its later amendments or comparable ethical standards.

Informed consent Informed consent was obtained from all individual participants included in the study.

\section{References}

1. Liang $\mathrm{W}$ et al (2020) Cancer patients in SARS-CoV-2 infection: a nationwide analysis in China. Lancet Oncol 21:335-337. https ://doi.org/10.1016/S1470-2045(20)30096-6

2. Yu J, Ouyang W, Chua MLK, Xie C (2020) SARS-CoV-2 Transmission in patients with cancer at a Tertiary Care Hospital in Wuhan, China. JAMA Oncol. https://doi.org/10.1001/jamao ncol.2020.0980

3. Grasselli G et al (2020) Baseline characteristics and outcomes of 1591 patients infected with SARS-CoV-2 admitted to ICUs of the Lombardy Region, Italy. JAMA. https://doi.org/10.1001/ jama.2020.5394

Publisher's Note Springer Nature remains neutral with regard to jurisdictional claims in published maps and institutional affiliations. 